\title{
Development in Nimbus Mattress
}

\author{
T.Manoj Prasath, R.Kishore Kanna, R.Vasuki
}

\begin{abstract}
The Nimbus proficient framework is a definitive weight redistribution sleeping cushion in the Nimbus run ,offering propelled dynamic treatment and all out patient administration though couldn't care less situations. Exchanging weight surfaces have been appeared to diminish the episodes of weight bruises contrasted and standard emergency clinic bedding and weight decreasing surfaces. This has been created by new therapeutic gadget directions and is demonstrated for the treatment of patients with all evaluations of weight bruises and for counteractive action in patients who are at extremely high danger of creating weight injuries. A propelled sleeping cushion supplanting framework with exchanging weight redistribution. The radiance framework consolidates programmed alterations of cell weights.
\end{abstract}

Rundown phrases-Bedding, Regulator Air Cells, ,Pressure Redistribution.

\section{INTRODUCTION}

Glow 3 and Nimbus 3 Professional Dynamic Flotation Systems for the anticipation, treatment and the executives of weight ulcers[1].

Radiance 3 and Nimbus 3 Professional frameworks involve a siphon and sleeping pad substitution which can be utilized on standard medical clinic and typical household beds. Beds can be balanced or profiled with the sleeping pad in position[2].

The Nimbus 3 Professional sleeping pad has the accompanying extra highlights to empower the patient to be proned and to help with weight zone and patient consideration the board:

- A Head Section Deflate Control to enable the three head cells to be completely flattened.

- Individual Vent Valves to enable 16 of the 20 cells to be freely emptied.

The Nimbus 3 and Nimbus 3 Professional sleeping cushions fuse an propelled Auto Matt sensor cushion which ensures that the patient is consequently bolstered at ideal weights paying little heed to estimate, stature, position or weight circulation. On the off chance that heart failure happens, the Nimbus 3 and Nimbus 3 Professional sleeping pads can be emptied in under 10 seconds to enable cardiovascular revival techniques to be performed.

Revised Manuscript Received on July 18, 2019.

T.Manoj Prasath, Department of Biomedical Engineering, BIHER, Chennai- 600073, Tamil Nadu, India.

R.Kishore Kanna, Department of Biomedical Engineering, BIHER, Chennai- 600073, Tamil Nadu, India.

Dr.R.Vasuki Department of Biomedical Engineering, BIHER, Chennai- 600073, Tamil Nadu, India.

\section{II.MATERIAL AND METHODOLOGY \& TEST RESULTS}

The Aura 3 Professional sleeping cushion has indistinguishable number of cells from the Nimbus 3 bedding ( 20 cells). The capacity of the initial four cells at the head end of the sleeping pad is diverse on the Nimbus 3 Professional[3]:

The three cells in the Head Section are either completely expanded or completely emptied, contingent upon the situation of the Head Deflate Control, to help with patient consideration the board. The cells are exceptionally controlled to empower them to be completely collapsed.[4]

The controls and markers are situated on the front board, and a complex caution framework separates between typical task and real framework issues. In the event that a caution circumstance is distinguished a glimmering marker will light up, together with a sign of the reason for alert, and a capable of being heard cautioning will sound. [5]

The siphon can be fixed to the foot end of a medical clinic bed by the different bed section. The bed section fits in the siphon handle and afterward cuts onto most normal bed outlines. The siphon can likewise be remained on the floor, either upstanding or on its back spread.

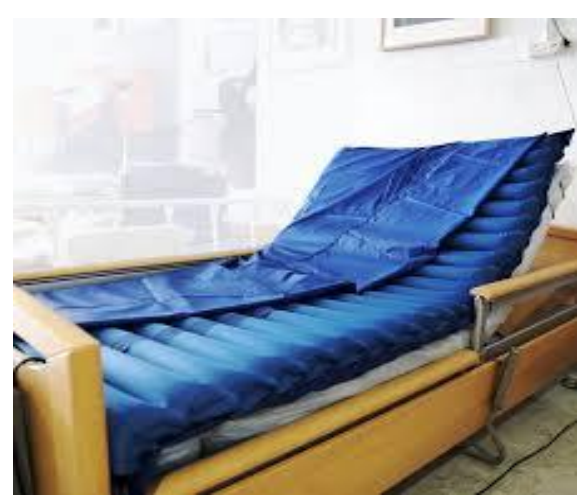

\section{FUTURE WORK}

Stage 1:All the segments required for the development of the Nimbus Mattress has been purchased.The vital papers and articles required for the improvement of the task has been finished amid the principal period of the venture[6].

Future Work:All the segments required for the development of the respiratory Nimbus Mattress has been purchased.Theoretically the gadget is demonstrated, essentially the gadget is yet to be designed.Further the methodology must be pursued and the Mattress is to be built. 


\section{REFERENCES}

1. DiBenedetto RJ, Nguyen AV. Weight Redistribution: an appreciated resurgence and a request for alert. Chest 2008; 111:1482-3.

2. Keith RL, Pierson DJ. Entanglements of air cells. Abedside approach. Clin Chest Med 2009;17:439- 51.

3. Herlich A. Complexities from verifying the troublesome aviation route. Int Anesthesiol Clan 2009;35:13-30..

4. Jokic R, Zintel T, Sridhar G, Gallagher CG, Fitzpatrick MF. nimbuss bedding reactions to head way and leg way in relatives of patients with the weight hypoventilation disorder. Thorax 2009; 55: 940-5.

5. Teichtahl H. The heftiness hypoventilation disorder returned to. Chest 2010;120:336-9. 